\title{
A Discrete Lattice Model of Quasi-brittle Fracture in Porous Graphite
}

\author{
Craig N Morrison ${ }^{* 12}$, Mingzhong Zhang ${ }^{1}$, Andrey P Jivkov ${ }^{1}$, John R Yates ${ }^{1}$
}

\begin{abstract}
Lattice models allow the incorporation of length scale dependent micro-structural features and damage mechanisms into analyses of the mechanical behaviour of materials. We describe our 3D lattice implementation and its use in fracture simulations. The method is particularly suitable for modelling fracture of nuclear graphite. This is a quasi-brittle material in which there is considerable non-linearity prior to final fracture due to the inherent porosity which triggers a field of local distributed failures upon mechanical and thermal loading.

Microstructure representative models are generated with experimentally measured particle and pore size distributions and volume densities in two graphite grades. The results illustrate the effect of distributed porosity on the emerging stress-strain response and damage evolution. It is shown how the failure mode shifts from graceful, plastic-like, behaviour associated with substantial energy dissipation via distributed damage at lower porosities, to glass-like behaviour with negligible energy dissipation at higher porosities. Thus the work proposes a microstructure-informed methodology for integrity assessment of aging structures, where porosity increase is driven by environmental factors, such as radiation of nuclear graphite components.
\end{abstract}

\section{Keywords}

Nuclear graphite; Porosity; Brittle-ligament Lattice; Damage evolution; Quasi-brittle behaviour

\section{Introduction}

Nuclear-grade graphite has been used as a fast neutron moderator in over 100 reactors throughout the world [1], with planned use in the Generation IV Very High Temperature Reactor. A main advantage of graphite over other moderator materials, such as light and heavy water, is its suitability for use as a structural material. As a result, developing a means of predicting the structural integrity of graphite under complex loading and in demanding environments such as a nuclear reactor core is critical for predicting plant lifetime and in-service performance.

Nuclear graphite has a feature-rich multiphase microstructure consisting of petroleum or pitch coke filler particles distributed within a matrix of binder material; usually coal-tar pitch. Distributed within both the matrix and filler phases is a population of porosity. This population can be broadly grouped into 3 main categories[2]:

\footnotetext{
${ }^{1}$ Mechanics and Physics of Solids Research Group, Modelling and Simulation Centre, The University of Manchester, Oxford Road, Manchester M13 9PL, UK

${ }^{2}$ Nuclear FiRST Doctoral Training Centre

*craig.morrison@manchester.ac.uk
} 
- Gas evolution cracking - During manufacture liquid pitch is impregnated into the graphite to increase density. In the subsequent baking of the green article the pitch boils resulting in the evolution of gas through the matrix phase [3].

- Calcination cracks - During the heating and cooling procedures involved in calcinations, the variation of the thermal expansion/shrinkage of the two solid phases of graphite's microstructure induce cracks $[2,4]$.

- Mrozowski cracks - These form for similar reasons to calcination cracks during the graphitization process $[2,4]$.

These flaws can vary in length scale, ranging from the $\mathrm{nm}$ scale to $\mathrm{mm}$ scale depending on the grade of graphite in question. The variation of input materials and manufacturing processes strongly influences the resulting microstructure so that different grades of graphite can have widely different properties.

The work in this paper focuses on two grades of graphite for potential use in future generations of high temperature reactors. IG110 is a fine-grained graphite of isotropic macroscopic properties which is currently in use in the Japanese High Temperature Test Reactor (HTTR), which first reached criticality in November 1998 [5]. It also remains a possibility for Generation IV high temperature reactors. Another possibility for high temperature reactors is PGX - a medium grained semi-isotropic graphite. The use of these two grades allows an effective comparison of the proposed methodology for grades of varying pore and particle sizes.

Component-scale failure of graphite has been shown to be dependent on its discrete multiphase microstructure, with the primary failure mechanism being the coalescence of micro-cracks into a critically sized flaw [6]. Such a response is considered to be a property of the class of quasi-brittle materials, allowing graphite to be considered alongside rock, concrete and cement in its failure behaviour. Distinctive properties of quasi-brittle materials include a reduced stiffness upon loading prior to failure and a residual load carrying capacity beyond the peak load [7]. The initial softening appears similar to plasticity although the individual phases remain brittle.

Failure modelling of quasi-brittle materials has led to the development of a number of local fracture models, whereby mechanistic understanding at the length scale of microstructural features referred to as the meso-scale in this context - can be incorporated into component scale models [8]. In this way the macro-scale dependence on these microstructural failure mechanisms can be recreated in a physically realistic manner.Most current local approaches, such as weakest link (WL) assumptions, are phenomenological, with parameters calculated by curve fitting to macroscopic experimental data[9]. Such approaches do not account for the chain of events leading to macroscopic failure, from micro-crack nucleation, through micro-crack interaction and to coalescence, observable at the microscale of quasi-brittle materials [10].

A continuum-based approach, intended to account for micro-crack nucleation and growth, is the cohesive zone modelling $[9,11]$, where the failure initiation and local softening in the quasi-brittle materials are represented by special cohesive elements. The advantage of this approach is that it is easily incorporated into the existing finite element formulations, and indeed cohesive elements are offered as standard in most finite element packages. However, the cohesive zone modelling relies on the introduction of cohesive elements, hence the modelling predefines the potential crack paths. 
Further, the formulation of the constitutive behaviour of the cohesive elements is phenomenological, involving calibration against observed macroscopic behaviour. Microstructureinformed models, such as the one offered in this work, have the potential to assist in deriving mechanistic constitutive laws for cohesive zone modelling.

Discrete approaches offer a promising method of developing material constitutive equations based on the actual micromechanical processes occurring. Lattice models form a branch of local discrete models, wherein a lattice is formed through the connection of nodes via discrete elements including springs [12] and beams [13]. The designation of element properties as representative of the micromechanical failure mechanisms can allow a realistic macro-mechanical response. Unlike WL methods, where bond failure signals total sample failure, lattice models form a statistically parallel system. This allows redistribution of load once a bond is broken amongst the remaining bonds.

Lattice models have been successfully developed for graphite $[4,14]$ and concrete $[13,15]$. Previous models have been limited in their ability to reproduce a full range of Poisson's ratio for elastic materials. Regular 2D beam-lattices using hexagonal unit cells, such as [10], can only be used for an isotropic elastic material with Poisson's ratio below $1 / 3$ and $1 / 4$ in plane stress and plane strain, respectively [15]. This is sufficient for most of the materials classified as quasi-brittle. However, the evolution of damage via micro-crack formation and growth is intrinsically a 3D phenomenon and physically realistic studies require 3D lattices. Regular 3D lattices, essential for realistic fracture modelling, suffer the same problems. Simple cubic lattices [16] and more complex face-centred cubic (FCC) and hexagonal closely-packed (HCP) lattice arrangements [17] have been demonstrated to be suitable only for materials with a Poisson's ratio of 0 [18]. The 3D lattice used in this work offers a significant improvement, as it can represent any isotropic material with Poisson's ratio of up to $1 / 4$, similarly to $2 \mathrm{D}$ lattices in plane strain, as shown in next section.

The work extends the site-bond 3D lattice model developed by Jivkov and Yates [19]. Sites at the centre of unit cells are connected to neighbouring sites by bonds. Previous work on this model using beam elements as bonds have demonstrated its ability to represent isotropic elastic materials with Poisson's ratio between -1 and 0.5 [19]. One of the issues with using beam elements is that no unique correspondence between the discrete and the continuum response of a cell can be established without the use of generalised continuum theory [19]. Therefore, in the current work bundles of linear springs are used to replace the beam elements as bonds.

This paper presents a methodology for construction of a meso-scale site-bond 3D lattice accounting for experimentally measured particle and pore size distributions as an extension on previous work [20]. A parametric study between a surface-based damage parameter and a standard mechanical damage parameter under uni-axial tension is used to establish a relationship between the volume fraction of distributed pores and progressive mechanical damage. It is demonstrated how the increase of porosity due, for example, to radiation-induced microstructure changes, embrittles the material, which can provide a scientific underpinning for integrity assessment of aging graphite components.

\section{Theory and Model}

The site-bond model uses a regular tessellation of space into truncated octahedral cells as a representation of the material microstructure, Fig. 1(a). This choice is dictated by the observation 
that the truncated octahedron is the regular shape closest to the average cell in Voronoi tessellations of real microstructures, in terms of faces, edges and vertices [21]. Hence, our cellular representation of the microstructure can be considered as an initial topological homogenisation. The truncated octahedron comprises of six square faces, normal to principal axes, and eight hexagonal faces, normal to the octahedral axes. The cellular lattice is used to maintain link to the microstructure features to be accounted for in the model. In addition, a discrete lattice of sites located at cell centres, and bonds connecting neighbouring sites, is generated for computational purposes. As a result, each site is connected to its neighbours by 14 bonds, six in the principal directions, $B_{1}$, with lengths equal to the cell size in principal direction, $L$, and eight in the octahedral directions, $B_{2}$, with lengths equal to $\sqrt{ } 3 / 2 L$ Fig. $1(b)$.

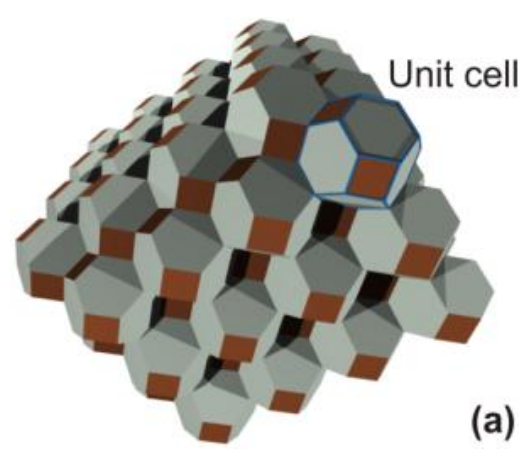

(a)

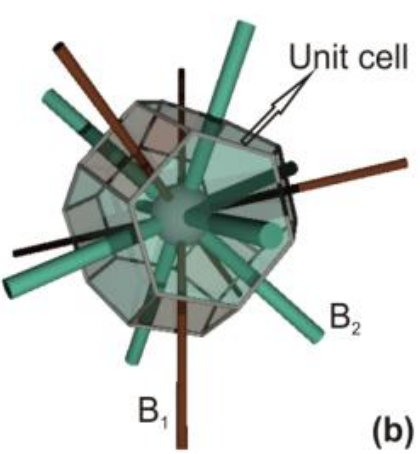

(b)

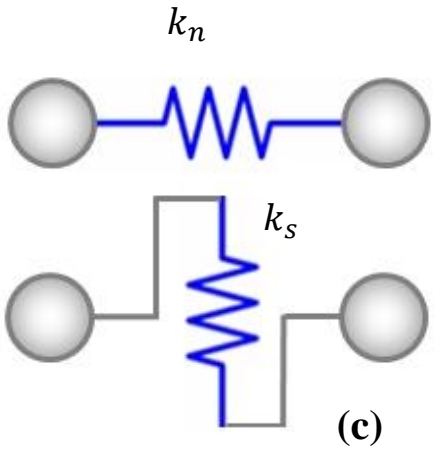

(c)

Fig. 1. (a) The cellular site-bond lattice with a single highlighted unit cell; (b) unit cell with bonds; (c) Normal and shear springs

In this site-bond model the bonds between sites are modelled as bundles of elastic springs. Generally, a bundle should contain six springs to represent the six possible degrees of freedom between sites, three translational/linear springs (one normal/axial and two shear) and three rotational/angular springs (one twist/axial and two bending). From a mechanical perspective, the linear and the angular springs should resist the symmetric (strains in continuum) and the skewsymmetric (rotations in continuum) parts of the local displacement gradient, respectively. Mathematically, these two tensors are fully uncoupled, meaning that no relation between linear and angular spring behaviour can be established. The linear springs can be calibrated using the correspondence between classical continuum strain energy in the cell and in the springs under identical displacement fields; this is explained below. However, the angular springs cannot be resolved without recourse to a generalised continuum theory, such as [22], and the corresponding material length-scale required in such a theory. Experimental work is planned to establish the validity of the generalised continuum description and the relation between the material length-scale and real microstructure length-scales.

A recent paper considered the bonds to be represented by bundles of linear springs only; with one normal and two shear springs per bond [23] as shown in Fig. 1(c). In such a case, and assuming the two shear springs in a bundle to have equal stiffness, there are four spring constants to be calibrated using energy equivalence between the discrete and continuum cells: $k_{n}^{p}$ and $k_{s}^{p}$-normal and shear stiffness in principal direction; $k_{n}^{o}$ and $k_{s}^{o}$ - normal and shear stiffness in principal direction. It has been shown analytically that such a configuration represents a case of macroscopic cubic elasticity, 
with three elastic constants describing the material behaviour [23]. The resulting indeterminacy, with three elastic constants available for calibration of four spring stiffness constants, could be solved in a number of ways. It has been suggested that the shear stiffness in the principal direction $k_{s}^{p}$ be set to zero [23]. The local behaviour is not affected by this decision, because shear on planes parallel to the principal directions is also resisted by the shear and normal springs in the octahedral direction. Furthermore it has been shown that for an isotropic material [23], with the selection $k_{s}^{p}=0$, the remaining spring stiffnesses in this model are related to the elastic modulus, $E$, the Poisson's ratio, $v$, and the cell size, $L$, by:

$$
k_{n}^{p}=\frac{E L}{4(1+v)(1-2 v)} \quad k_{n}^{o}=\frac{(1+2 v) E L}{4(1+v)(1-2 v)} \quad k_{s}^{o}=\frac{(1-4 v) E L}{4(1+v)(1-2 v)}
$$

It can be seen that the shear stiffness $k_{s}^{o}$ will become negative when Poisson's ratio $v$ exceeds $1 / 4$. This indicates that the site-bond model with springs can represent isotropic materials with Poisson's ratio in the range $-1<v \leq 1 / 4$. The result differs from the analysis using beams [19] and the reason is that the angular springs have been neglected in [23] and in the current work, while the beams introduce specific coupling between bending and shear.

The spring stiffnesses for the models of the two graphite grades, IG110 and PGX, were evaluated using Eq. 1 and the macroscopic material properties shown in The cell size calculation is described in the next section.

Table 1, with data obtained from [24] and [25] respectively. The cell size calculation is described in the next section.

Table 1. Material properties for PGX and IG110 graphite. Data or IG110 from Ref. [23]; Data for PGX from Ref. [24]

\begin{tabular}{c|cc}
\hline & IG110 & PGX \\
\hline$E(\mathrm{MPa})$ & 9800 & 8300 \\
$v$ & 0.14 & 0.11 \\
\hline
\end{tabular}

\section{Distribution of Material Features}

Statistical distributions of filler particle and pore sizes for the two grades of nuclear graphite in the virgin state were generated according to experimental data from recent works by Kane et al. [26]. A lognormal distribution was used to reproduce the particle distribution parameters. The pore distributions were produced using third-order polynomial parameters specified in [26]. Basic parameters for the particle and pore size distributions and volume fractions for the two grades in their virgin state are given in Table 2.

Table 2. Particle and pore size parameters reproduced from experimental data.

\begin{tabular}{c|cc}
\hline & IG110 & PGX \\
\hline Mean particle diameter $(\mu \mathrm{m})$ & 27 & 92 \\
Mean pore area $\left(\mu \mathrm{m}^{2}\right)$ & 98 & 197.9 \\
Particle volume fraction & $20 \%$ & $20 \%$
\end{tabular}


The procedure to construct the site-bond lattice with a suitable meso length scale is as follows:

- A cellular structure contained in a region is specified in terms of number of cells in the three principal directions. Thus for $\mathrm{Nx}, \mathrm{Ny}, \mathrm{Nz}$ cells in the three directions, the cellular structure will contain $C=\mathrm{Nx}^{*} \mathrm{Ny} * \mathrm{Nz}+(\mathrm{Nx}-1) *(\mathrm{Ny}-1) *(\mathrm{Nz}-1)$ cells.

- Particles with assumed spherical shape are assigned to all cells, with one particle per cell with random size from the experimental particle size distribution.

- A representative cell length, $L$, is calculated using Eq. 2, using the volume of the cellular structure as a function of cell length, Eq. 3 , the total volume of the distributed particles and the particle volume fraction $\theta$

$$
\begin{aligned}
& L=\sqrt[3]{\frac{2 \sum_{i=1}^{C} v_{\text {particle }}^{i}}{C \theta}} \\
& V_{\text {lattice }}=C \frac{L^{3}}{2}
\end{aligned}
$$

Such an assignment can be used to assess how representative is a cellular structure with $C$ cells for prescribed particle size distribution and volume density. If the scatter in calculated cell length for different random assignments of particles in $C$ cells is smaller than some value, then the cellular structures with $\mathrm{C}$ cells can be accepted as sufficiently representative. Fig. 2 illustrates a sensitivity study of the length scale dependence on the number of cells. With this result, and accepting a maximum standard deviation of 6.02 and 27.9 ( 4 and 5\% of the mean cell length) for IG110 and PGX respectively, we justify the use of a $15 \times 15 \times 15$ cellular structure in the subsequent simulations as a compromise between accuracy and computational expense. This structure contains 6119 cells and 39046 bonds.

(a) IG110

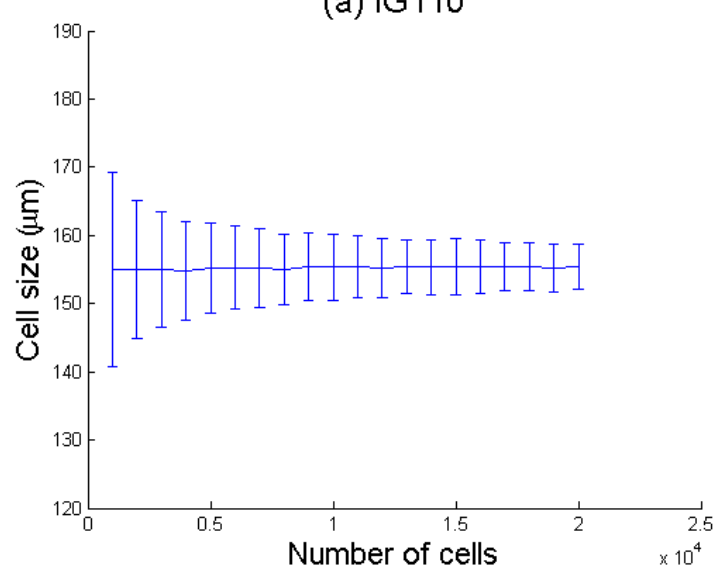

(b) PGX

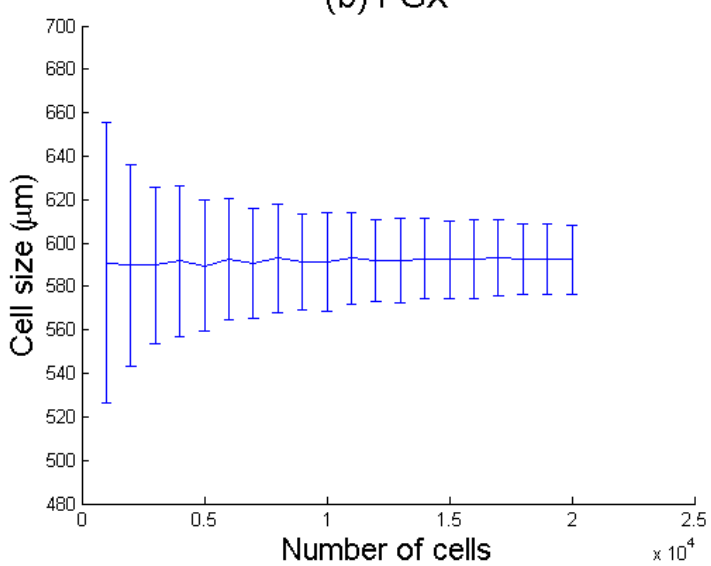

Fig. 2. The sensitivity of the produced cell size from a sample from a given population for varying number of cells for (a) IG110 graphite (b) PGX graphite. Error bars indicate one standard deviation for 1000 different samples of particle size distributions. 
In the paper by Kane et al. [26], it was observed that, at the obtained image resolution, less than $1 \%$ of the pores present in both grades of graphite occurred within the filler particles. Hence, it is reasonable to assume in our porosity modelling procedure that all porosity occurs in the matrix phase of graphite, and we can ignore the calcination and Mrozowski cracks. Furthermore, as with the particles, the pores are assumed to be spherical.

Following lattice construction and length scale calculation, pores are distributed to the faces between cells. Multiple pores can be allocated to each cell face in order to reach the desired pore volume fraction (porosity). This is done in a succession of passes over the cell faces in a random manner. An illustration of distributed porosity on cell faces, equivalently bond centres, is given in Fig. 3. Multiple pores per face are not shown for clarity of the figure.

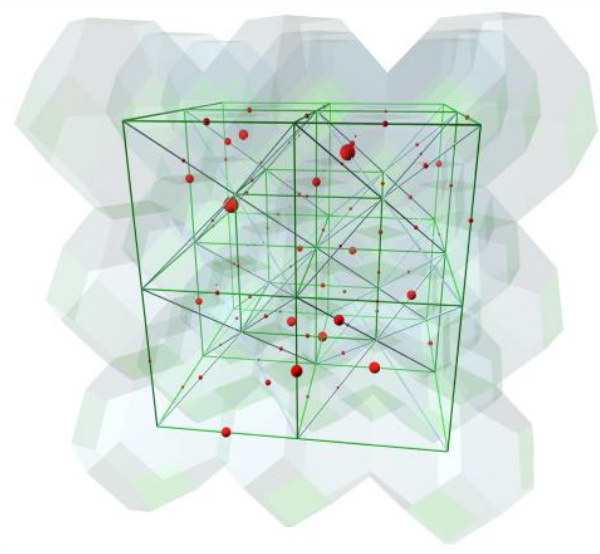

Fig. 3. A visual representation of the site bond model with pores distributed on faces between cells.

Multiple lattices were generated for the two grades of graphite for varying pore volume fractions from the virgin state up to a maximum of $60 \%$, with the increase in volume fraction giving an insight into the effects of increased porosity over a nuclear reactor lifetime on damage. It is assumed that as the porosity increases both the particle and porosity statistical size distributions remain the same.

Mechanical damage upon loading is represented by progressive failure of the bonds in the site-bond lattice. The behaviour of the two types of springs is shown in Fig. 4. The shaded areas represent the energy dissipated upon failure of the spring, $G_{c}$, which is different for different bonds, depending on the assigned pores. The energy is calculated with $G_{c}=\gamma A_{c}$, where $\gamma$ is the energy lost in creation of two surfaces, and $A_{c}$ is the intact area of the face, i.e. the original face area minus the cross-section area of all pores assigned to the face. The material parameter $\gamma=9.7 \mathrm{~J} / \mathrm{m}^{2}$ is taken from the literature as the enthalpy for creation of two surface in graphite [27]. Notably, this is the energy of separation of ideal atomic lattice of graphite and should not be compared to fracture energy directly. Therefore, this value is identical for the two graphite grades analysed here. The fracture energy of a particular grade is an emergent property from the underlying energy of separation and its microstructure features and distribution. It can be represented by the area under the simulated stress-strain curve and will be different for different failure modes, e.g. normal or shear failure. The pore areas allocated to each face are the maximum pore cross sectional areas. This is justified to a large extent in the context of the discrete computational model where the pore can be placed anywhere between the sites and have the same failure effect on the spring bundle. Thus, $G_{c}$ tends towards the maximum spring failure energy (material surface energy) for pore size approaching zero, 
and tends towards zero for pore size approaching face area. Bonds are removed prior to simulations if the initial pore area exceeds the area of the face on which it is situated.
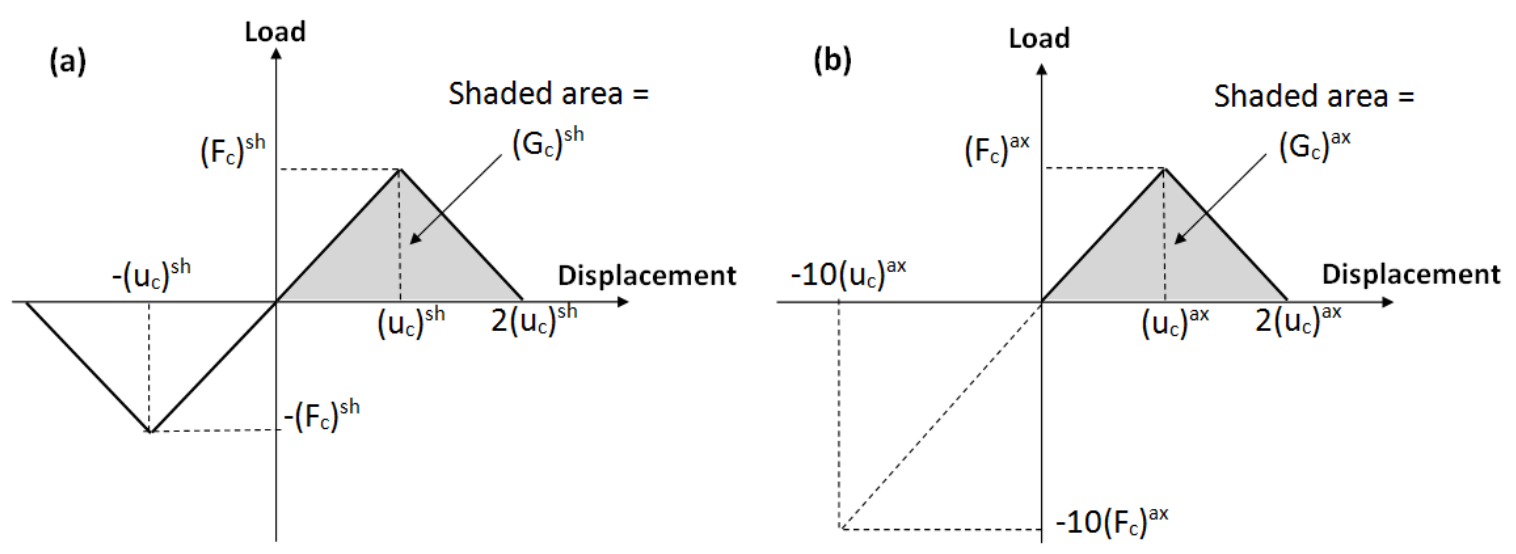

Fig. 4. Failure criteria for (a) shear springs and (b) axial springs

The assumption used for these criteria is that if the spring damage initiates at a critical relative displacement, then the damage evolution is linear (softening branch) and terminates with spring failure at two times the critical displacement. This is a reasonable assumption for local brittle failure and allows for direct calculation of the critical displacement and force from knowledge of the failure energy and initial spring stiffness. Notably, the axial springs are not allowed to fail in compression, and the behaviours of the two types of springs are uncoupled in the current model. Equal failure energies for the axial and shear springs in a bond have been used.

\section{Results and Discussion}

Sample site-bond lattices with distributed porosity, with a range of volume fractions, for each grade of graphite were loaded under displacement control in uni-axial tension. The simulations were performed in steps of increasing boundary displacements until the final rupture of the samples. This was an automated solution, such that multiple spring failures could potentially occur within a loading increment. While this differs from previous works $[17,27]$, where the load increment was driven by individual failures, it represents the behaviour that would be observed when testing real samples. At each increment end, the macroscopic stress was determined from the reaction forces on the boundary with prescribed displacements and the physical area of this boundary; the macroscopic strain was determined from the current displacement level and the length of the sample. As the strains and stresses are small within the loading range to failure for this material, the obtained engineering strain and stress are approximately equal to the true values.

Data for the models analysed is given in Table 3. This includes the model length scale derived from each specific particle distribution to cells, the fraction of initially removed bonds due to excessive pore size on corresponding faces, and the initial elastic moduli determined prior to damage initiation in the lattice. Notably, the elastic moduli, given in Table 1 and used for the spring stiffness calibration, were derived with specimens containing virgin state porosities. This is the reason for the difference between the virgin state elastic moduli in Tables 1 and 3. In principle, the model can be calibrated without difficulties to account for given porosity (missing bonds) and provide required macroscopic elastic modulus. However, the initial calibration of the full lattice is preferable here to 
facilitate comparison between cases with increasing porosity. It also helps to note that the porosity effect on the elastic modulus is not linear and arises from the specific combination of a particle size distribution (via the length scale) and a pore size distribution. For example, compare the difference between the virgin states of the two grades where the higher porosity grade, PGX, is stiffer than the lower porosity grade, IG110, relative to the benchmark values in Table 1, because of the different relation between the cell size (particle sizes) and the pore sizes.

Table 3. Basic parameters of the analysed models: cell size derived from particle distribution to cells; fraction of removed bonds due to large pores on faces; elastic modulus of voided model prior to damage.

\begin{tabular}{c|cccc}
\hline & $\begin{array}{c}\text { Pore volume } \\
\text { fraction } \\
\text { (porosity) }\end{array}$ & $\begin{array}{c}\text { Cell size } \\
(\mu \mathrm{m})\end{array}$ & $\begin{array}{c}\text { Fraction of } \\
\text { bonds } \\
\text { removed }\end{array}$ & $\begin{array}{c}\text { Initial } \\
\text { Young's } \\
\text { modulus } \\
\text { (MPa) }\end{array}$ \\
\hline IG110 & 0.1473 (virgin) & 148.4 & 0.026 & 9474.6 \\
& 0.3 & 159.1 & 0.086 & 8417.6 \\
\hline PGX & 0.6 & 148.8 & 0.319 & 5083.0 \\
\hline & 0.2149 (virgin) & 607.0 & 0.002 & 8377.4 \\
& 0.4 & 547.0 & 0.268 & 4938.7 \\
\hline
\end{tabular}

Fig. 5 illustrates the emergent stress-strain curves for both grades of graphite with different porosities. The initial linear behaviour in all cases is governed by corresponding macroscopic elastic modulus, listed in Table 3. Beyond the linear response, clear quasi-brittle non-linearity prior to the fracture point is observed in Fig. 5 for cases with low porosities (below 30\%). For higher pore volume fractions, very little non-linearity is exhibited - the samples failed in strictly brittle manner at marginally higher strains than for the cases with lower porosity. The last points of each stress-strain curve correspond to final failure, i.e. the separation of the modelled volume into disjoint regions. Further insight into these behaviours is gained by considering the evolution of the mechanical damage.
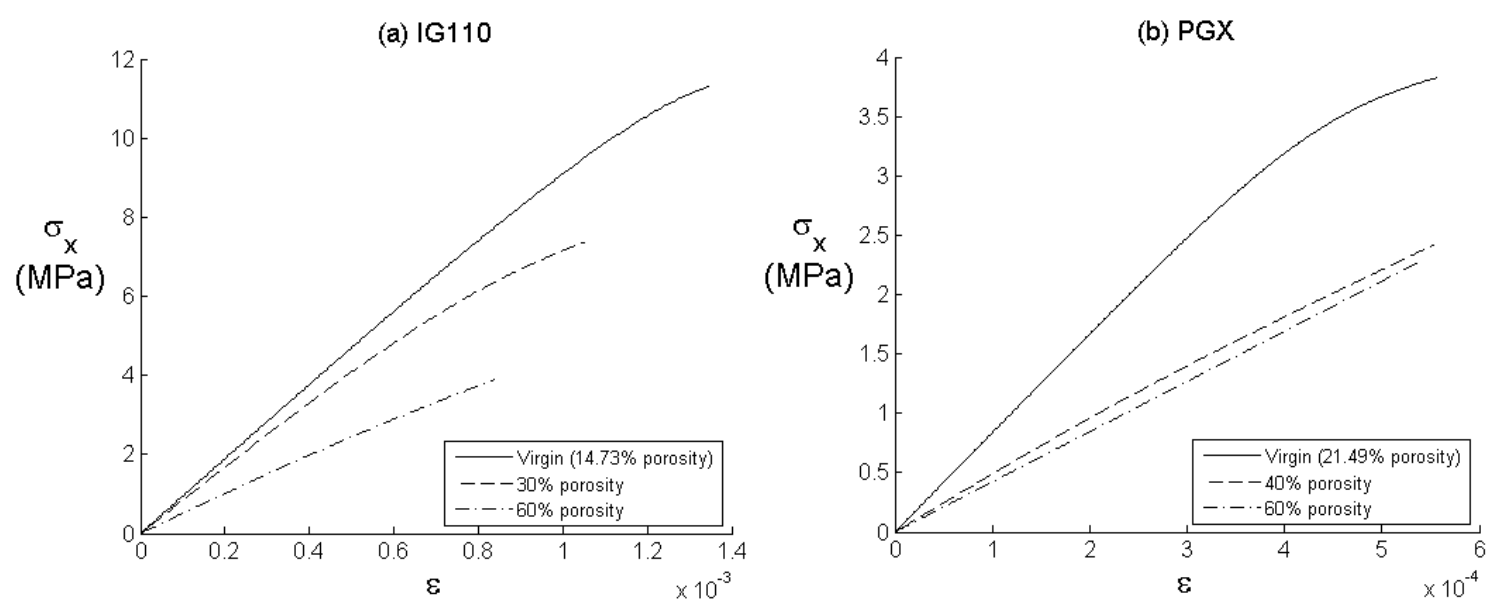

Fig. 5. The stress-strain response under of uni-axial tension of (a) IG110 graphite (b) PGX graphite 
Fig. 6 shows the development of the mechanical damage parameter $D_{E}$ with increasing applied strain. The damage parameter $D_{E}$ is defined as a relative change of the material's elastic modulus:

$$
\mathrm{D}_{\mathrm{E}}=1-\frac{\mathrm{E}}{\mathrm{E}_{0}}
$$

It can be seen for IG110 graphite, Fig. 6(a), at relatively low values of applied strain $(<0.03)$ the $D_{E}$ for all porosity volume fractions is increasing, with a greater rate of increase for higher porosity volume fractions. This is an expected behaviour and suggests non-linearity in the corresponding stress-strain curves, Fig. 6(a), which is not clearly identifiable for all curves due to the scales used. The damage evolution in IG110 continues with increasing rate at the virgin state; with increasing but smaller rate at $30 \%$ porosity; but with decreasing rate at $60 \%$ porosity prior to failure. Similar damage evolution behaviour is observed in PGX, Fig. 6(b), where however, the $40 \%$ porosity exhibits decreasing damage evolution rate before failure, and the $60 \%$ porosity exhibits a negligible increase of $D_{E}$ before failure.
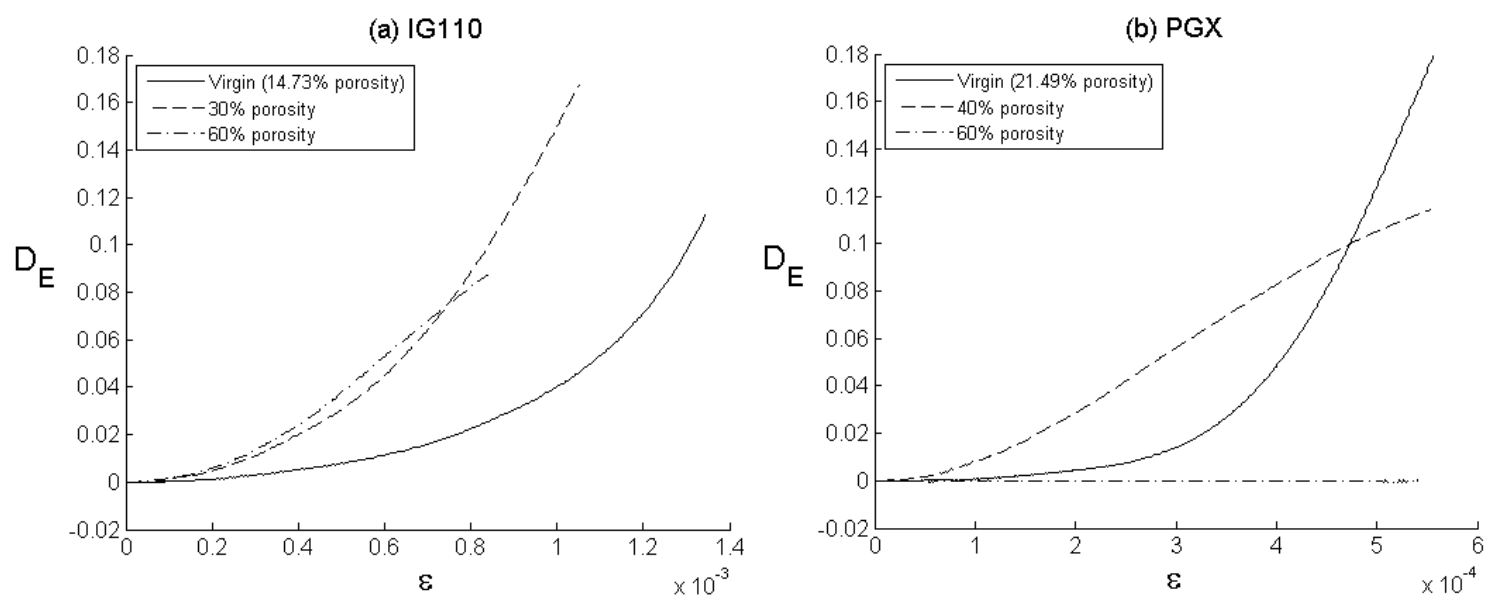

Fig. 6 . The variation of the mechanical damage parameter $D_{E}$ with applied strain.

The observed behaviour of decreasing damage evolution rates at higher porosities suggest that the graphite grades studied exhibit "avalanche" behaviour, typical of purely brittle failure. The bonds remaining in the corresponding models continue to carry the load until the sudden simultaneous rupture of all bonds required for sample disintegration. This is particularly pronounced in PGX at $60 \%$ porosity where practically no mechanical damage developed prior to final failure. The avalanche behaviour is potentially the result of the large number of pores required on each bond to reach the high pore volume fractions. Consequently the accumulated pore size and from there the failure strength of all bonds is approximately equal, specifically in 60\% porosity PGX. This results in a simultaneous rapture of a bond population critical for the sample integrity. Although the result that higher porosity embrittles the material may seem counter-intuitive, it correlates with the observations that irradiation causes porosity increase and reduction of fracture toughness [29].

These observations require further analysis of the developing micro-crack population following the procedure outlined in [28]. Specifically, it is necessary to develop understanding and quantify the relation between failed area and its topology (surface-based damage measure), and the emerging mechanical damage. Nevertheless, with this pilot study we have established a methodology for 
microstructure-informed prediction of the fracture energy of graphite. This can be calculated as the area under the stress-strain curve for particular microstructure, which includes all energy loss via distributed micro-cracking and the eventual avalanche-like rapture, divided by the sample area. A more precise quantification of the surface-based damage would improve the fracture energy estimates. Current results show that the increase of porosity reduces the "gracefulness" of the graphite behaviour with corresponding reduction of the fracture energy, which can be used for integrity assessment of aging components, where environmental factors cause porosity increase.

The predicted failure stress in the virgin states can be compared to the limited data available for these grades [30]. The comparison shows that our predictions are half of the mean experimental values reported. The reason for this discrepancy could be the assumption for the spring behaviour shown in Fig. 4, where a large softening branch has been introduced. This was done by considering that the graphite matrix contains a system of nano-pores, which could act as a lower-scale softening mechanism. On the other side the selected separation energy of the matrix does not include the presence of nano-porosity. These options require further consideration. With the current separation energy the results suggest that the spring behaviour should be assumed with negligible softening, in which case the stress values shown in Fig. 5 would roughly double.

One point to be noted is that in the present work the increase of porosity has not been accompanied by any change in the statistical pore size distribution. In some cases this could be unrealistic. However, the model provides the means to calculate the failure energy should changes in size distribution and volume density of pores be measured experimentally. In addition, the results presented here are specific to uniaxial loading and hence useful for prediction of fracture energy. The methodology, however, can be used to derive damage evolution laws for incorporation in continuum-base finite element analysis of cracked components. It has been previously shown that under more complex stress states, such plane stress existing ahead of a crack front, the evolution of damage results in an elastic anisotropy [28]. Derivation of a damage tensor for such loading cases under the proposed methodology will be a subject of further publications.

The presented model for elastic-brittle local behaviour can be extended to elastic-plastic or viscoelastic behaviour prior to spring failure initiation with the use of non-linear springs and dampers. This has been done for other lattice models [31] and is a subject of ongoing work for the lattice proposed here.

The approach developed here aimed at linking microstructure features to macroscopic response. It is demonstrated how a macroscopic property, e.g. stress-strain curve or damage, emerges from the underlying microstructure and the separation energy of defect-free graphite atomic lattice. The presented results are specific to the uniaxial tension of a volume element of a limited size. These allow for derivation of cohesive zone behaviour and fracture toughness associated with the particular loading considered. One important extension of the work will consider different deformation conditions, specifically those present ahead of a macroscopic crack. This way the model could be used for the analysis of the fracture process zone size. The parameters controlling this size are still not clearly understood. According to Aliha and Ayatollahi [32], Awaji et al [33] and Claussen et al. [34], the size of the fracture process zone scales with the squared ration of the fracture toughness and tensile strength. However, Ayatollahi and Aliha [35] have pointed out that the size is related to the average grain size alone. The modelling of the entire macro-crack tip region with 
microstructure-informed lattice will require a significant computational effort. As a first step into understanding the different damage evolutions within the macro-crack tip region, we have planned simulations with representative volume elements subjected to the deformation conditions at a number of spatial positions. The results of such simulations will be analysed to estimate the need for large-size lattice covering the entire macro-crack tip region and reported in future communications. Before completing the planned programme, it is difficult to estimate the effects of grain, pore and cell sizes on the macroscopic fracture behaviour of graphite.

\section{Conclusions}

A procedure to construct a meso-scale site-bond lattice model is described that accounts for experimentally measured particle and pore size distributions and volume fractions. The procedure is particularly suitable for the analysis of the collective behaviour of micro-cracks, which is the precursor for the non-linear behaviour in quasi-brittle materials, such as graphite.

For the graphite grades investigated, the predicted stress-strain behaviour at low porosities, in virgin state and up to $30 \%$, is typical of a quasi-brittle material. The failure is preceded by a significant dissipation of energy by distributed micro-cracking. This is reflected in the growth of the macroscopic mechanical damage.

At higher porosities, the predicted stress-strain behaviour shifts to a typical brittle, characterised by sudden failure with reduced or negligible energy dissipation from distributed micro-cracking. The failure is an avalanche-like event, whereas a population of bonds critical to the component integrity fail simultaneously.

The procedure can therefore be used to estimate the changes in material fracture energy as a function of the changes in the microstructure with aging. Three areas requiring further investigation are identified: (1) provision of experimental evidence for the behaviour of graphite as a generalised continuum at the particle length scale and the corresponding calibration of angular springs in bond bundles; (2) quantitative analysis of micro-crack population to relate surface-based damage to mechanical damage for prediction of fracture energy; (3) analysis of complex stress sates to derive damage evolution laws for continuum finite element analysis.

\section{Acknowledgements}

CN Morrison greatly appreciates the support from EPSRC via Nuclear FiRST Doctoral Training Centre. $M$ Zhang and AP Jivkov acknowledge the support from EPSRC via grant EP/J019763/1, "QUBE: QuasiBrittle fracture: a 3D experimentally-validated approach", and from BNFL for the Research Centre for Radwaste \& Decommissioning. JR Yates acknowledges support from EDF R+D.

\section{References}

[1] IAEA, "Characterization, Treatment and Conditioning of Radioactive Graphite from Decommissioning of Nuclear Reactors," 2006. 
[2] M. R. Bradford and A. G. Steer, "A structurally-based model of irradiated graphite properties," J. Nucl. Mater., vol. 381, no. 1-2, pp. 137-144, Oct. 2008.

[3] M. R. Joyce, T. J. Marrow, P. Mummery, and B. J. Marsden, "Observation of microstructure deformation and damage in nuclear graphite," Eng. Fract. Mech., vol. 75, no. 12, pp. 36333645, Aug. 2008.

[4] E. Schlangen, P. E. J. Flewitt, G. E. Smith, a. G. Crocker, and A. Hodgkins, "Computer Modelling of Crack Propagation in Porous Reactor Core Graphite," Key Eng. Mater., vol. 452-453, pp. 729-732, Nov. 2010.

[5] M. Ogawa and T. Nishihara, "Present status of energy in Japan and HTTR project," Nucl. Eng. Des., vol. 233, no. 1-3, pp. 5-10, Oct. 2004.

[6] M. Mostafavi and T. J. Marrow, "Quantitative in situ study of short crack propagation in polygranular graphite by digital image correlation," Fatigue Fract. Eng. Mater. Struct., vol. 35, no. 8, pp. 695-707, Aug. 2012.

[7] A. Hodgkins, T. J. Marrow, M. R. Wootton, R. Moskovic, and P. E. J. Flewitt, "Fracture behaviour of radiolytically oxidised reactor core graphites: a view," Mater. Sci. Technol., vol. 26, no. 8, pp. 899-907, Aug. 2010.

[8] J. Lemaitre, "Local approach of fracture," Eng. Fract. Mech., vol. 23, pp. 523-537, 1986.

[9] R. de Borst, "Fracture in quasi-brittle materials: a review of continuum damage-based approaches," Eng. Fract. Mech., vol. 69, pp. 95-112, 2002.

[10] Z. P. Bažant and S. Pang, "Activation energy based extreme value statistics and size effect in brittle and quasibrittle fracture," J. Mech. Phys. Solids, vol. 55, no. 1, pp. 91-131, Jan. 2007.

[11] Z. Bažant, "Concrete fracture models: testing and practice," Eng. Fract. Mech., vol. 69, pp. 165-205, 2002.

[12] A. Pazdniakou and P. M. Adler, "Lattice Spring Models," Transp. Porous Media, vol. 93, no. 2, pp. 243-262, Feb. 2012.

[13] E. Schlangen and E. Garboczi, "Fracture simulations of concrete using lattice models: computational aspects," Eng. Fract. Mech., vol. 57, no. 2, pp. 319-332, 1997.

[14] N. N. Nemeth and R. L. Bratton, "Overview of statistical models of fracture for nonirradiated nuclear-graphite components," Nucl. Eng. Des., vol. 240, no. 1, pp. 1-29, Jan. 2010.

[15] D. Griffiths and G. Mustoe, "Modelling of elastic continua using a grillage of structural elements based on discrete element concepts," Int. J. Numer. Methods Eng., vol. 50, pp. 1759-1775, 2001.

[16] E. Schlangen, "Crack Development in Concrete, Part 2: Modelling of Fracture Process," Key Eng. Mater., vol. 385-387, pp. 73-76, 2008.

[17] Y. Wang and P. Mora, "Macroscopic elastic properties of regular lattices," J. Mech. Phys. Solids, vol. 56, no. 12, pp. 3459-3474, Dec. 2008. 
[18] A. P. Jivkov, D. L. Engelberg, R. Stein, and M. Petkovski, "Pore space and brittle damage evolution in concrete," Eng. Fract. Mech., vol. In press, 2012.

[19] A. P. Jivkov and J. R. Yates, "Elastic behaviour of a regular lattice for meso-scale modelling of solids," Int. J. Solids Struct., vol. 49, no. 22, pp. 3089-3099, 2012.

[20] C. N. Morrison, A. P. Jivkov, G. Smith, and J. R. Yates, "Lattice-spring modeling of graphite accounting for pore size distribution," Key Eng. Mater., vol. In press.

[21] S. Kumar, S. K. Kurtz, J. R. Banavar, and M. G. Sharma, "Properties of a three-dimensional Poisson-Voronoi tesselation: A Monte Carlo study," J. Stat. Phys., vol. 67, no. 3-4, pp. 523551, May 1992.

[22] A. R. Hadjesfandiari and G. F. Dargush, "Couple stress theory for solids," Int. J. Solids Struct., vol. 48, no. 18, pp. 2496-2510, Sep. 2011.

[23] M. Zhang, C. N. Morrison, and A. P. Jivkov, "A meso-scale site-bond model for elasticity: Theory and calibration," Mater. Res. Innov., vol. In press, 2013.

[24] Y. Kaji, W. Gu, M. Ishihara, T. Arai, and H. Nakamura, "Development of structural analysis program for non-linear elasticity by continuum damage mechanics," Nucl. Eng. Des., vol. 206, no. 1, pp. 1-12, May 2001.

[25] T. T. C. Products, "Special Graphite and Compound Material Products." .

[26] J. Kane, C. Karthik, D. P. Butt, W. E. Windes, and R. Ubic, "Microstructural characterization and pore structure analysis of nuclear graphite," J. Nucl. Mater., vol. 415, no. 2, pp. 189-197, Aug. 2011.

[27] J. Abrahamson, "The surface energies of graphite," Carbon N. Y., vol. 11, no. 4, 1973.

[28] A. P. Jivkov, "Structure of micro-crack population and damage evolution in concrete," in 13th International Conference on Fracture, Beijing, China, 2013, pp. 1-10.

[29] C. Berre, S. L. Fok, B. J. Marsden, P. M. Mummery, T. J. Marrow, and G. B. Neighbour, "Microstructural modelling of nuclear graphite using multi-phase models," J. Nucl. Mater., vol. 380, no. 1-3, pp. 46-58, Oct. 2008.

[30] M. Ishihara, J. Sumita, T. Shibata, T. lyoku, and T. Oku, "Principle design and data of graphite components," Nucl. Eng. Des., vol. 233, no. 1-3, pp. 251-260, Oct. 2004.

[31] G. S. O'Brien, "Discrete visco-elastic lattice methods for seismic wave propagation," Geophys. Res. Lett., vol. 35, no. 2, p. L02302, Jan. 2008.

[32] M. R. M. Aliha and M. R. Ayatollahi, "Analysis of fracture initiation angle in some cracked ceramics using the generalized maximum tangential stress criterion," Int. J. Solids Struct., vol. 49, no. 13, pp. 1877-1883, Jun. 2012.

[33] H. Awaji, T. Matsunaga, and S.-M. Choi, "Relation between Strength, Fracture Toughness, and Critical Frontal Process Zone Size in Ceramics," Mater. Trans., vol. 47, no. 6, pp. 1532-1539, 2006. 
[34] N. Claussen, B. Mussler, and M. V. Swain, "Grain-Size Dependence of Fracture Energy in Ceramics," Commun. Am. Ceram. Soc., vol. 65, no. 1, pp. C14-16, 1982.

[35] M. R. Ayatollahi and M. R. M. Aliha, "Fracture Analysis of Some Ceramics Under Mixed Mode Loading," J. Am. Ceram. Soc., vol. 94, no. 2, pp. 561-569, Feb. 2011. 